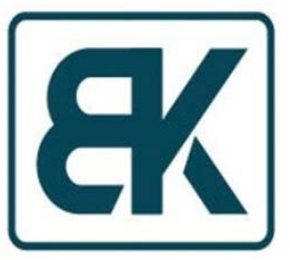

\title{
Antiviral Drugs
}

By Megha Agrawal, PhD

Co Editor-in-Chief

Biotechnology Kiosk, 2, 3 (2020)

DOI: https://doi.org/10.37756/bk.20.2.3.1

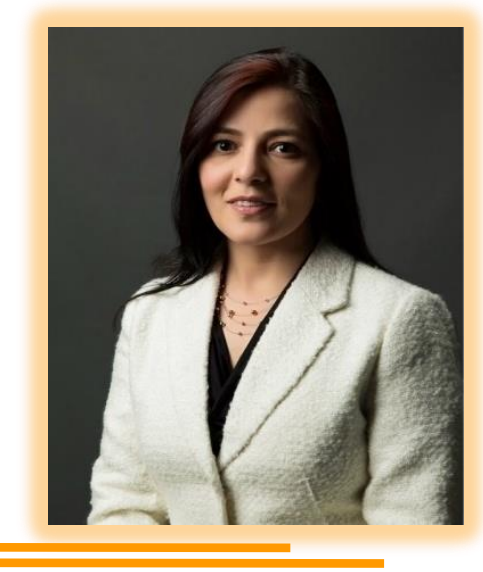

\section{Can Antiviral Drugs Stop Coronavirus (COVID-19)?}

\section{Preventing Coronavirus Spread}

The recent emergence of a novel, pathogenic coronavirus known as Severe Acute Respiratory Syndrome Coronavirus 2 (SARS-CoV-2, officially COVID-19) in China and its subsequent rapid spread across the world has put global health at risk. Some early research data suggest that the cell entry of coronaviruses depends on the process of binding of the viral spike (S) proteins to cellular receptors along with $S$ protein priming by host cell proteases as depicted in Figure 1 [1]. Further, the latest understanding tells us that angiotensin-converting enzyme 2 (ACE2) is the cellular receptor for SARS coronavirus and the new coronavirus, SARSCoV-2, which is causing the epidemic COVID-19 [2]. Current research has indicated the need to focus more on understanding the cellular factors that are used by SARS-CoV-2 for entry that could provide vital insights into viral transmission and subsequently lead to reveal therapeutic targets [1].

Previous studies have suggested that members of the family Coronaviridae can circulate in the human population that results in mild respiratory disease [3]. However, the serious forms of respiratory disease such as the SARS-CoV and the Middle East respiratory syndrome coronavirus (MERSCoV) occur via transmission from animals to humans and that can cause severe respiratory diseases [1]. In view of the rapid spread of coronavirus, a global effort is currently initiated that is focused on developing vaccines and also identifying other effective treatment approaches that employ drug agents with demonstrated antiviral activity against SARSCoV and MERS-CoV or related positive-sense RNA viruses [4]. To this end, researchers demonstrated the effectiveness of Camostat mesilate for its protection mechanisms against COVID-19 [1]. Figure 1 illustrates how SARS-CoV-2 uses the SARS-CoV receptor ACE2 for entry and the serine protease TMPRSS2 for $S$ protein priming [1, 4]. Recent data using drug agents demonstrated that a TMPRSS2 inhibitor which is approved for clinical use can block entry. This might pave the way for an effective treatment option (Figure 1) [1]. 


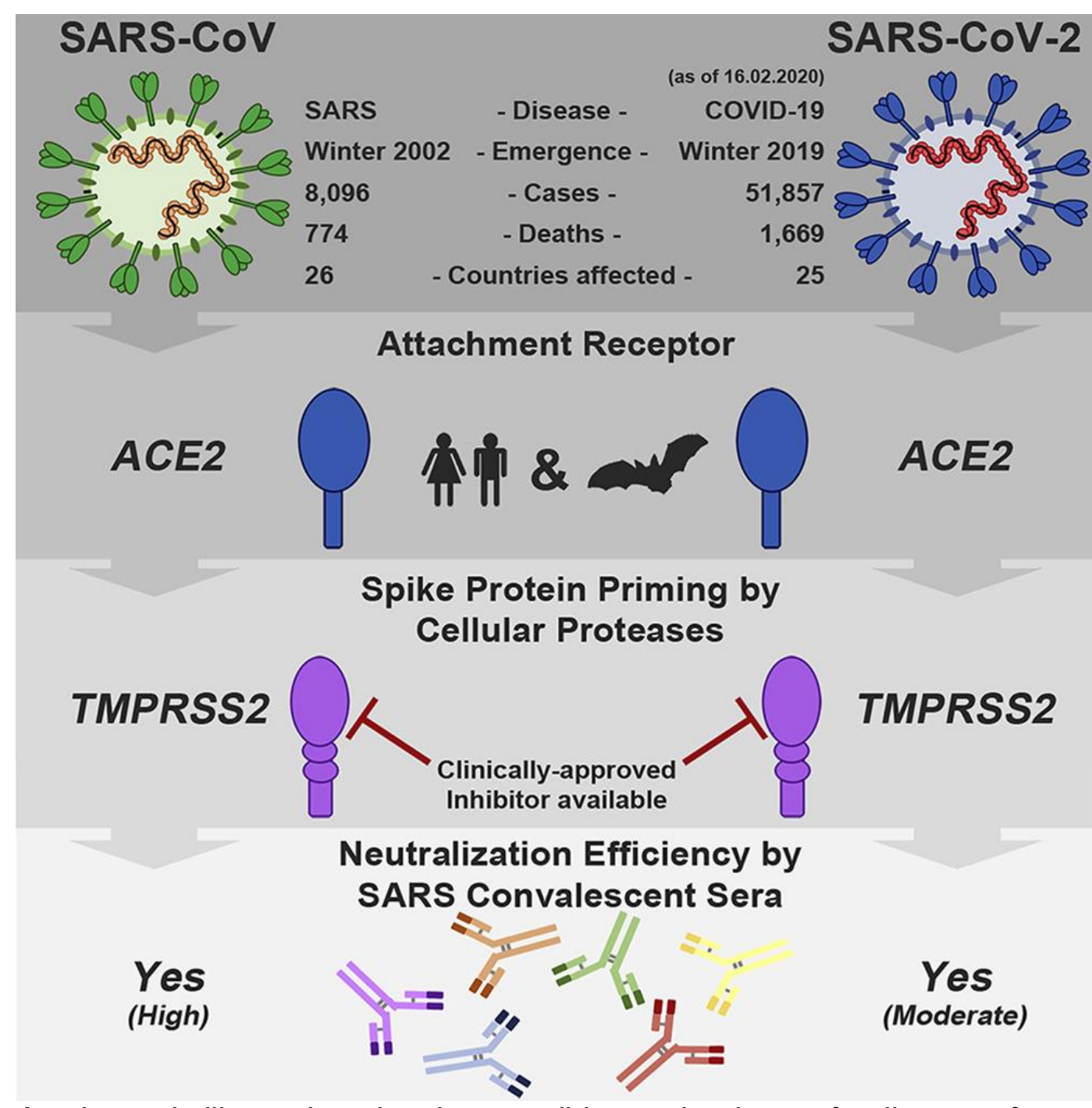

Figure 1: A schematic illustration showing possible mechanisms of cell entry of coronaviruses and the therapeutic actions of inhibitor [Source: Cell (2020)].

\section{Repurposing Drugs to Battle Coronavirus}

The latest preclinical data shown by researchers that uses the nucleotide analogue Remdesivir (RDV) are shown to be promising to combat coronavirus [4]. It has been shown that the RDV compound exhibit a broad range of antiviral activities against several RNA viruses [5-7], and also in battling SARS-CoV and MERS-CoV [4]. Some other studies have shed light on the effectiveness of RDV for its active actions against coronaviruses with divergent RNAdependent RNA polymerases (RdRp) [4, 8]. The optimistic view of the drug remdesivir indicates that this compound might work for the pathogen responsible for COVID-19. However, there are not enough biochemical data available yet that can support these findings and also provide a possible mechanism of action [4].

This pre-clinical study done on the compound RDV has suggested that the 
compound works by inhibiting an enzyme known as an RNA-dependent RNA polymerase. This polymerase is used by many RNA viruses including coronaviruses to replicate themselves [4]. In contrast, other RNA viruses that are known as retroviruses (HIV) use an enzyme called reverse transcriptase. This subsequently creates DNA from an RNA blueprint. This implies that the antiviral drug compound RDV has significant potential to be effective since coronaviruses also use RNA-dependent enzymes [4].

In this study, researchers coexpressed the MERS-CoV nonstructural proteins nsp5, nsp7, nsp8, and nsp12 (RdRp) in insect cells. This was chosen as a part of a polyprotein to study the mechanism of inhibition of MERS-CoV RdRp by RDV [4]. The triphosphate form of the inhibitor (RDVTP) was shown to be competing with its natural counterpart ATP [4]. Based on the data obtained, researchers explained the high potency of RDV against RNA viruses in cell-based assays [4]. Researchers also showed that the sera from patients having convalescent SARS could be used to crossneutralize SARS-2-S-driven entry. It was also shown that once the drug was incorporated into the growing RNA chain, the virus no longer replicated. These early results are promising that reveal key similarities between SARS-CoV-2 and SARS-CoV infection and that can be leveraged to identify a potential target for antiviral intervention [4].

\section{Concluding Remarks}

The early pre-clinical trials using antiviral drugs have shown promise in blocking COVID-19. Further, latest breakthroughs on structure-based rational design of binders with enhanced affinities to either ACE2 or the $S$ protein of the coronaviruses can be leveraged for the development of vaccines. All these steps are believed to lead to the suppression of viral infection of coronavirus.

\section{References for Further Reading}

1. Markus Hoffmann; Hannah Kleine-Weber; Simon Schroeder; Nadine Krüger; Tanja Herrler; Sandra Erichsen; Tobias S. Schiergens; Georg Herrler; Nai-Huei Wu; Andreas Nitsche; Marcel A. Müller; Christian Drosten; Stefan Pöhlmann, SARS-CoV-2 Cell Entry Depends on ACE2 and TMPRSS2 and Is Blocked by a Clinically Proven Protease Inhibitor, Cell 181 , $1-10$ (2020), https://doi.org/10.1016/i.cell.2020.02.052

2. Renhong Yan, Yuanyuan Zhang, Yaning Li, Lu Xia, Yingying Guo, Qiang Zhou, Structural basis for the recognition of the SARS-CoV-2 by full-length human ACE2, Science

(2020), http://dx.doi.org/10.1126/science.abb250 $\underline{7}$

3. Corman V.M. Lienau J. Witzenrath M., Coronaviruses as the cause of respiratory infections, Internist (Berl.). 2019; 60: 1136-1145, DOI: $\underline{10.1007 / s 00108-019-}$ $\underline{00671-5}$

4. Calvin J Gordon, Egor P Tchesnokov, Joy Y. Feng, Danielle P Porter and Matthias Gotte, The antiviral compound remdesivir potently inhibits RNA-dependent RNA polymerase from Middle East respiratory syndrome coronavirus, Journal of 
Biological Chemistry, (2020); DOI: 10.1074/jbc.AC120.013056.

5. Siegel, D., Hui, H. C., Doerffler, E., Clarke, M. O., Chun, K., Zhang, et. al., Discovery and Synthesis of a Phosphoramidate Prodrug of a Pyrrolo[2,1-f][triazin-4amino] Adenine C-Nucleoside (GS-5734) for the Treatment of Ebola and Emerging Viruses. Journal of medicinal chemistry 60 , (2017), 1648-1661, https://doi.org/10.1021/acs.jmedchem.6b $\underline{01594}$

6. Lo, M. K., Jordan, R., Arvey, A., Sudhamsu, J., Shrivastava-Ranjan, P., Hotard, A. L., Flint, M., McMullan, L. K., Siegel, D., Clarke, M. O., Mackman, R. L., Hui, H. C., Perron, M., Ray, A. S., Cihlar, T., Nichol, S. T., and Spiropoulou, C. F. GS-5734 and its parent nucleoside analog inhibit Filo-, Pneumo-, and
Paramyxoviruses, Scientific reports 7 , 43395,

https://doi.org/10.1038/srep43395

7. Warren, T. K., Jordan, R., Lo, M. K., Ray, A. S., Mackman, R. L., et. al., Therapeutic efficacy of the small molecule GS-5734 against Ebola virus in rhesus monkeys. Nature 531, 381-385, (2016), https://doi.org/10.1038/nature17180

8. Brown, A. J., Won, J. J., Graham, R. L., Dinnon, K. H., 3rd, Sims, A. C., Feng, J. Y., Cihlar, T., Denison, M. R., Baric, R. S., and Sheahan, T. P. Broad spectrum antiviral remdesivir inhibits human endemic and zoonotic deltacoronaviruses with a highly divergent RNA dependent RNA polymerase, Antiviral research 169, 104541, (2019), DOI: 